\title{
CLIC TEST FACILITY DEVELOPMENTS AND RESULTS
}

\author{
R.ÊBossart, H.ÊBraun, F.ÊChautard, M.ÊComunian, J.P.ÊDelahaye, J.C.ÊGodot, I.ÊKamber, J.H.B.ÊMadsen, L.ÊRinolfi, \\ S.ÊSchreiber, G.ÊSuberlucq, I.ÊWilson and W.ÊWuensch, \\ CERN, 1211 Geneva 23, Switzerland
}

\section{INTRODUCTION}

The objectives of the CLIC Test Facility (CTF) are to study the generation of short intense electron bunches using a laser driven photocathode in an RF gun, to generate $30 \hat{\mathrm{E} G H z} \mathrm{RF}$ power for high gradient tests of prototype CLIC components, and to test beam position monitors. The performance of the CTF has improved dramatically in the course of the past year and highlights are presented here. The layout of the CTF is shown in Fig. $\hat{E} 1$.

The RF gun now has a $\mathrm{Cs}_{2} \mathrm{Te}$ photocathode, enabling the use of the fourth harmonic of the YLF laser system (262Ênm). Laser pulse lengths down to 8Êps full-width-half height (FWHH) and energies of $0.5 \hat{E} \mathrm{~mJ}$ have been produced. The CTF operates with a repetition rate of $10 \hat{\mathrm{E} H z}$ with either single bunches or trains of up to 48 bunches. Trains are produced by splitting the laser pulse. The RF gun consists of a $11 / 2$ cell cavity, a photocathode, a focusing solenoid and a 4 cell booster cavity. The beam exits the gun with a momentum of $4.5 \hat{\mathrm{EMeV}} / \mathrm{c}$ and is then accelerated up to $92 \hat{\mathrm{EMeV}} / \mathrm{c}$ by the S-band travelling wave accelerating section. $30 \hat{\mathrm{E} G H z}$ power is generated when the beam is passed through the - un-powered - prototype CLIC main linac accelerating section [1]. The power is fed to the second prototype main linac accelerating section and the accelerating gradient produced in it is directly measured by reaccelerating the lead bunch of the drive train.

\section{PERFORMANCES}

In the 1994 run, the CTF produced $30 \hat{\mathrm{EGHz}}$ powers of up to $76 \hat{\mathrm{E} M W}$, which corresponds to a peak gradient of $123 \hat{\mathrm{E} M V} / \mathrm{m}$ in the $30 \hat{\mathrm{E} G H z}$ decelerating section and an accelerating structure. The highest $30 \hat{\mathrm{E} G H z}$ powers were average gradient of $94 \hat{\mathrm{E} M V} / \mathrm{m}$ in the $30 \hat{\mathrm{E} G H z}$ accelerating produced by a 48 bunch train with a total charge of $80 \hat{\mathrm{E} n C}$ section. Consistency between accelerating fields determined through RF power measurement and reacceleration was confirmed up to $76 \hat{\mathrm{E} M V} / \mathrm{m}$. There has never been any sign of RF breakdown in either accelerating section, any $30 \hat{\mathrm{E} G H z}$ component or waveguide at any power level achieved so far. In addition, the output periodically loaded waveguide of a prototype transfer structure was tested to 60ÊMW without RF breakdown. These results show that CLIC can be operated at nominal field levels with little or no conditioning.

The maximum power achieved in the 1994 CTF run was almost a factor 2 higher than that achieved in the 1993 run [2]. This improvement is mainly due to an increased beam energy of $92 \hat{\mathrm{E} M e V}$ which reduces the detrimental effect of long range transverse wakefields in the decelerating section. A second modulator and klystron -allowed the generation of the extra $3 \hat{E} G H z$ power. Further improvement came from raising the number of bunches to 48 which increased the charge passing through the decelerating section. This was made possible by an upgrade of the laser pulse train generator. The train generator upgrade has also given the capability to vary the laser pulse lengths. A longer laser pulse length reduces the effect of space charge in the RF gun and has given a single bunch charge at the gun output to $35 \hat{\mathrm{E} n C}$. This charge is more than twice the previous CTF record. The electron bunch length at this charge was $\sigma_{\mathrm{z} \hat{\mathrm{E}}}=2.4 \hat{\mathrm{E} m m}$ and thus further improvement can be expected. The maximum charges achieved in the CTF are summarized in TableÊ 1 . The single bunch charge is limited by space charge effects in the gun and short range transverse wakefields in the $3 \hat{E} G H z$ structure. Multibunch charge at the RF gun exit is limited by the available laser energy. The downstream charge is further limited by long range transverse wakefields and chromatic effects due to beam-loading in the S-band transmitted through the $30 \hat{\mathrm{E} G H z}$ decelerating section. For this charge the measured bunch length was $\sigma_{\mathrm{z} \hat{\mathrm{E}}} \cup \hat{\mathrm{E}} 1 \hat{\mathrm{E}} \mathrm{mm}$ which corresponds to the resolution limit of the streak camera. 


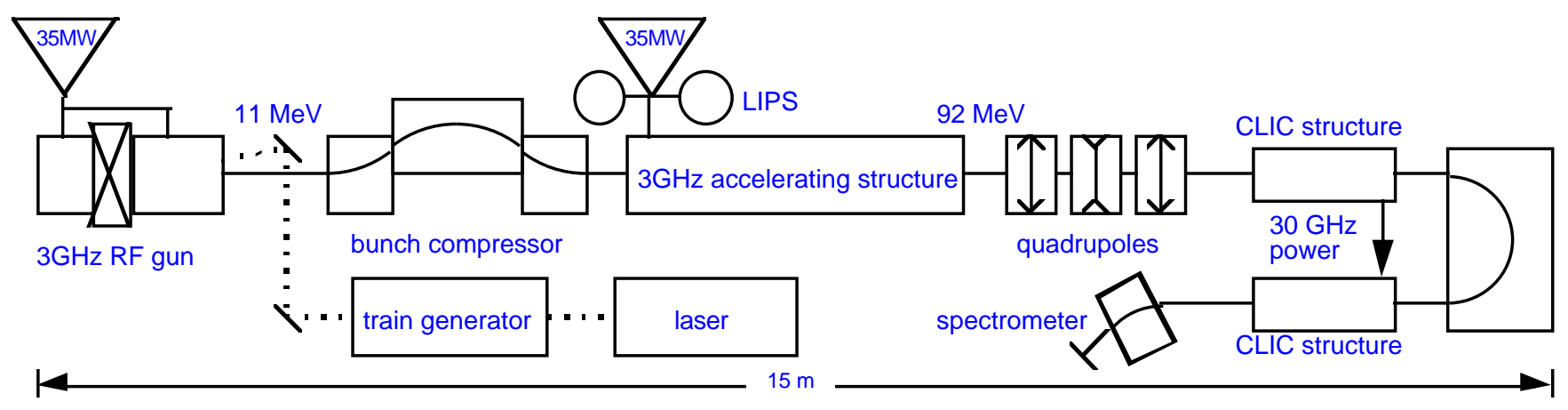

Figurê̂1: Layout of the CTF planned for 1995 


\begin{tabular}{|l|c|c|}
\hline position in beamline & $\begin{array}{c}\text { single bunch } \\
{[\mathrm{nC}]}\end{array}$ & $\begin{array}{c}48 \text { bunches } \\
{[\mathrm{nC}]}\end{array}$ \\
\hline RF Gun exit & 35 & 450 \\
3GHz structure exit & 20 & 160 \\
30GHz structure exit & 7 & 81 \\
\hline
\end{tabular}

TableÊ1: Maximum measured charges

\section{EMITTANCE MEASUREMENTS}

Emittance measurements were performed with single bunches by varying the strengths of two quadrupoles downstream of the $3 \hat{E} G H z$ structure and measuring the beam profiles on a transition radiation screen just upstream of the $30 \hat{E} G H z$ accelerating section. The measurement results together with simulation results from PARMELA are shown in Fig.ÊE2 Ê[3]. The normalized, $1 \hat{\mathrm{E}} \sigma$, rms emittance is used For these measurements the laser spot on the photocathode had a radius of $5 \hat{E} \mathrm{Em}$ and a duration of $8 \hat{\mathrm{E} p s}$ FWHH. The phase difference between the zero crossing of the electric field in the gun and the arrival of the laser pulse was 30r. Although the variation of emittance with bunch charge is qualitatively similar for measured and computed values, the measured emittances are systematically higher. This effect is not understood. The large error bars on measured emittances at high charges are caused by unstable beam conditions.

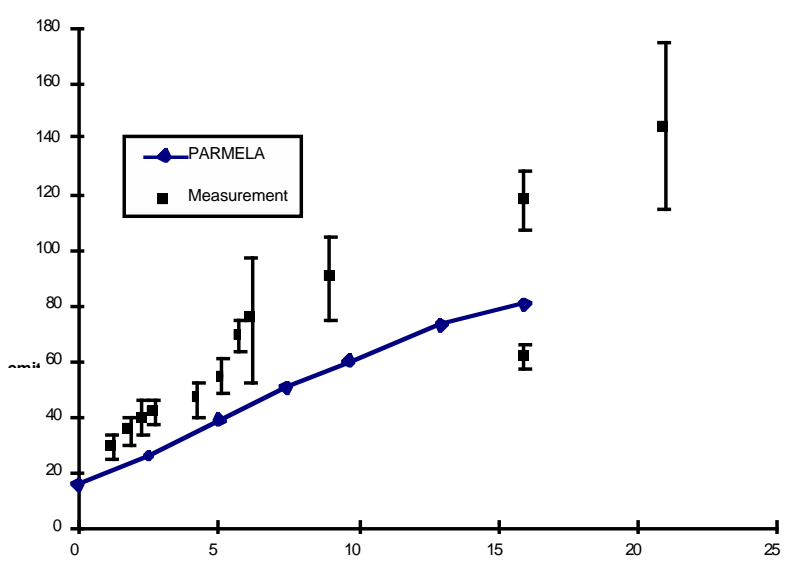

FigureÊ2: Emittance as a function of bunch charge

\section{RF PULSE COMPRESSION}

A: A phase jump of $180 \Upsilon 1.2 \hat{E} \mu$ s before the end of the RF pulse. This has been the standard mode of operation before the programmable phase shifter was available.

B: A phase jump of $+68 \Upsilon 1.2 \hat{E} \mu$ s before the end of the RF pulse, followed by a gradual phase shift from $+68 \Upsilon$ to +180 r during the remainder of the pulse.

C: A linearly decreasing phase by -30 during the first $3.3 \hat{E} \mu \mathrm{s}$, then 3 a jump of $+68 \Upsilon$, followed by a linear phase shift of $+112 \Upsilon$ during $1.2 \hat{\mathrm{E}} \mu \mathrm{s}$.

Scheme A produced a sharp rise followed by an exponential decay with an overshoot 2.5 times larger than the average pulse power. Scheme B delivered a nearly flat power pulse with an overshoot of only $20 \%$ above the average power. Nonetheless scheme B provided 10\% less acceleration of the beam than method A. This occurred because scheme B introduces a frequency shift of about $30 \hat{\mathrm{E} k H z}$ at the output of the LIPS cavities. This has been compensated in scheme $\mathrm{C}$ by the negative phase ramp at the beginning of the RF pulse. The energy gain of the beam with scheme $\mathrm{C}$ is $5 \%$ lower than scheme $\mathrm{A}$ for constant klystron power. Because the beam energy was not limited by klystron power but rather RF breakdowns in the $3 \hat{\mathrm{EGHz}}$ accelerating section the lower overshoot of scheme $\mathrm{C}$ is more important. An energy gain of $87 \hat{\mathrm{E} M e V}$ was achieved with scheme $\mathrm{C}$ and only $70 \hat{\mathrm{EMeV}}$ with scheme A.

\section{PHOTOCATHODES}

Nine photocathodes have been used in the RF gun during the $1994 \mathrm{CTF}$ run. Four $\mathrm{Cs}_{2} \mathrm{Te}$ cathodes were used at $100 \hat{\mathrm{E} M V} / \mathrm{m}$ for a total of 159 days. However, three others worked only at a lower field, $70 \hat{E} \mathrm{MV} / \mathrm{m}$, and were used for only a total of 22 days. The typical starting quantum efficiency (QE) was about 5\%, measured in a dc gun at $8 \hat{\mathrm{EMV}} / \mathrm{m}$. The QE was found to increase with increasing electric field during measurements with the photocathode in the RF gun, see Fig.Ê3.

The QE does not show a strictly exponential degradation with time. During a period of 4 to 5 days after installation in the RF gun, a rather fast decay with a 1/e

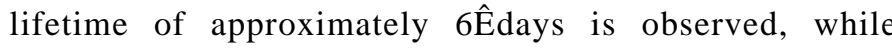
afterwards the QE decreases more slowly, with the 1/e decay time varying between 34 and 67 days for the next two months (the beam duty factor is typically $30 \%$ ).

The $3 \hat{E} G H z$ accelerating section is powered by a Measurements with closely spaced laser pulses have $35 \hat{E} M W$ klystron with a $4.5 \hat{E} \mu$ s long pulse compressed to demonstrated that the relaxation time of electrons in the $1.2 \hat{\mathrm{E}} \mu \mathrm{s}$ by two LIPS cavities as shown in Fig. $\hat{\mathrm{E}} 1$. This type of photocathode material is less than a few picoseconds. Two pulse compression requires a phase shift near the end of the klystron output pulse [4]. Using a new programmable $3 \hat{\mathrm{E} G H z}$ low level RF phase shifter, three phase shift schemes were tested. new photocathode materials which can be transported in air, unlike $\mathrm{Cs}_{2} \mathrm{Te}$ which requires a vacuum transfer system and preparation chamber, were tested. CsI with a thin layer of germanium has a QE of $0.19 \%$ at $100 \hat{\mathrm{EMV}} / \mathrm{m}$. A magnesium layer on a copper substrate has a $\mathrm{QE}$ of only $0.027 \%$ for the same electric field. 


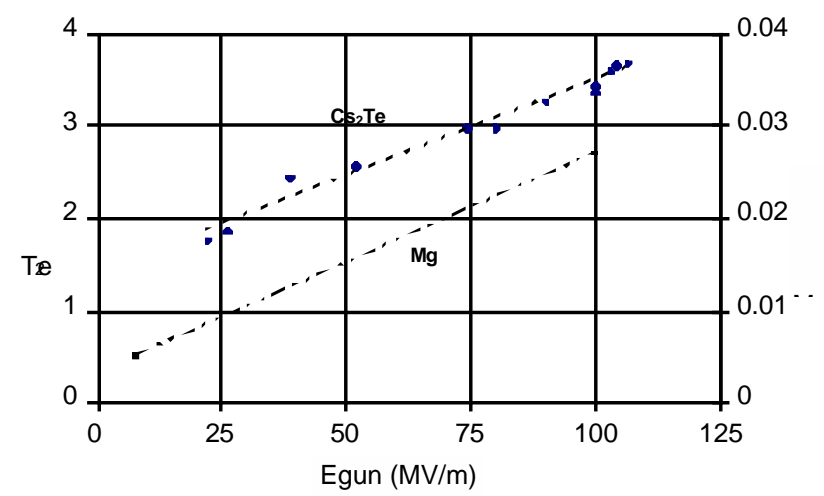

FigureÊ3: Quantum efficiency versus electric field for $\mathrm{Cs}_{2} \mathrm{Te}$ and $\mathrm{Mg}$ photocathodes.

\section{MODIFICATIONS FOR THE 1995 RUN}

In order to reduce the beam-loading and transverse wakefields in the $3 \hat{E} G H z$ accelerating section, the old spare LIL section used until now will be replaced by a high gradient, $1 \mathrm{~m}$ long structure borrowed from LAL [5].

A magnetic chicane bunch compressor between the RF gun assembly and the accelerating structure will be used in the 1995 run . An energy/phase correlation in a bunch (introduced by appropriate phasing of the booster cavity) together with the energy/path length dependence in the chicane compresses the bunch. The chicane consists of two $15 \hat{\mathrm{E}} \mathrm{cm}$ long left bending magnets and a $30 \hat{\mathrm{E}} \mathrm{cm}$ long right bending magnet [6]. Two quadrupoles upstream of the chicane and four downstream (not shown in Fig.Ê1) are used to match the beam in the transverse plane.

In order to increase the high charge performance of $\mathrm{CTF}$, a new RF gun is being constructed. A drawing of the RF geometry is shown in Fig. $\hat{E} 4$, and the main parameters are listed in TableÊ 2 . The design goals were to maximize aperture to allow a large beam radius, maximise acceleration in the first cell to keep the effect of space charge small, and to minimize the r/Q to minimize energy spread in bunch trains. These goals are achieved with a large iris aperture, a 10r concave cone around the cathode, and re-optimized cell lengths.

\begin{tabular}{|l|l|}
\hline number of cells & 3 \\
iris diameter [mm] & 40 \\
cone angle & $10 \Upsilon$ \\
frequency $[\mathrm{MHz}]$ & 2998.55 \\
output energy $[\mathrm{MeV}]$ & 6.58 \\
input power [MW] & 13.6 \\
max. field on photo cath. [MV/m] & 100 \\
\hline
\end{tabular}

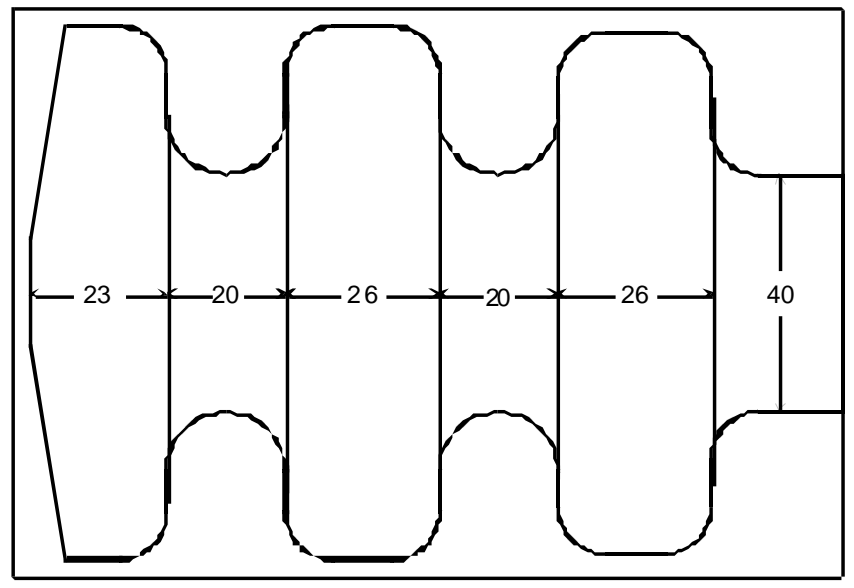

FigureÊ4: Sketch of the new RF gun (distances in $\mathrm{mm}$ )

\section{REFERENCES}

[1] H. Braun, R. Corsini, J.P. Delahaye, G. Guignard, C.D.ÊJohnson, J.H.B. Madsen, L. Thorndahl, I.ÊWilson, W.ÊWuensch, B.ÊZZotter, ÒCLIC - a compact and efficient high energy $\mathrm{e}^{+} / \mathrm{e}^{-}$Linear Collider,Ó this conference.

[2] R.Bossart, H. Braun, J.P.ÊDelahaye, K.K.ÊGeissler, J.C.ÊGodot, J.H.B.ÊMadsen, A.J.ÊRiche, L.ÊRinolfi, S.ÊSchreiber, S.ÊSladen, G.ÊSuberlucq, I.ÊWilson. W.ÊWuensch, ÒPerformances Obtained with the CERN Linear Collider Test Facility,Ó European Part. Acc. Conf., London, 1994, p. 680-682.

[3] M. Comunian, ÒEmittance Measurements in CTF,Ó Tesi per il corso di perfezionamento in Fisica, Universit di Padova, 1995.

[4] A. Fiebig and Ch. Schieblich, ÒA SLED Type Pulse Compressor with Rectangular Pulse Shape,Ó European Part. Acc. Conf., Nice, 1990, p. 937-939.

[5] G. Bienvenu and P. Brunet, ÒDark Current under Low and High Electric Field,Ó European Part. Acc. Conf., London, 1994, p. 775-777.

[6] F. Chautard, ÒLe compresseur de paquets dÕ/lectrons pour le banc de test du collisionaire lin/aire du CERNÓ, PhD-thesis in preparation, Paris University VI. 